\title{
Characterizing Dynamic Data Driven Applications Systems (DDDAS) in Terms of a Computational Model
}

\author{
Frederica Darema \\ National Science Foundation, Arlington, VA USA \\ darema@nsf.gov
}

\begin{abstract}
The DDDAS (Dynamic Data Driven Applications Systems) concept creates new capabilities in applications and measurements, through a new computational paradigm where application simulations can dynamically incorporate and respond to online field-data and measurements, and/or control such measurements. Such capabilities entail dynamic integration of the computational and measurement aspects of an application in a dynamic feed-back-loop, leading to unified SuperGrids of the computational and the instrumentation platforms. Examples of advances in applications capabilities enabled through DDDAS over traditional computational modeling methods, and advances in measurements methods, and instrumentation and sensor network systems, have appeared extensively in the literature. This paper concentrates in discussing a computational model representing the unified DDDAS computationmeasurement environments, and asymptotic cases leading to traditional computational environments, data assimilation, and traditional control systems.
\end{abstract}

Keywords: computational model, applications, measurements, dynamic runtime, sensors, grids.

\section{References}

1. NSF Workshop (March 2000), http: / / www. cise.nsf.gov/dddas

2. Douglas, C.C.: (2000-2009), http: / / www .dddas .org

3. Darema, F.: Grid Computing and Beyond: The Context of Dynamic Data Driven Applications Systems. In: Proceedings of the IEEE, Special Issue on Grid Computing (March 2005)

4. Darema, F.: Dynamic Data Driven Applications Systems: A New Paradigm for Application Simulations and Measurements. In: Bubak, M., van Albada, G.D., Sloot, P.M.A., Dongarra, J. (eds.) ICCS 2004. LNCS, vol. 3038, pp. 662-669. Springer, Heidelberg (2004)

5. Darema, F.: Dynamic Data Driven Applications Systems: New Capabilities for Application Simulations and Measurements. In: Sunderam, V.S., van Albada, G.D., Sloot, P.M.A., Dongarra, J. (eds.) ICCS 2005. LNCS, vol. 3515, pp. 610-615. Springer, Heidelberg (2005)

6. Darema, F.: Introduction to the ICCS 2006 Workshop on Dynamic Data Driven Applications Systems (2006) 
7. NSF Sponsored Workshop on DDDAS-Dynamic Data Driven Applications Systems, January 19-20 (2006), http: / /www. cise.nsf .gov/dddas

8. DDDAS-Dynamic Data Driven Applications Systems Program Solicitation, NSF 05-570, http: / /www.cise.nsf.gov/dddas

9. Darema, F.: Novel Drivers for Future InterNets: Dynamic Data Driven Applications Systems (DDDAS). In: 1st IEEE Workshop on Enabling Future Service-Oriented Internets GLOBECOM (2007)

10. Darema, F.: DDDAS Computational Model and Environments. Journal of Algorithms and Computational Technology (2009/2010) (to appear) 